\title{
Cronología del Estado Inca
}

VIRGILIO SCHIAPPACASSE F.

\section{RESUMEN}

Se contrastan sobre la base de un análisis estadístico y algunas consideraciones de naturaleza arqueológica, fechas de radiocarbono y de termoluminiscencia atribuidas al Horizonte Tardío o Inca de las áreas Centro Sur y Meridional andinas, supuestamente más antiguas que la cronología "histórica" generalmente aceptada, con la cronología propuesta independientemente por Adamska y Micheczynski (1996) y por Bauer (1992) para el núcleo del imperio. Se concluye que la cronología del Estado Inca es todavía un problema en discusión que requiere de un mayor acopio de fechados, constituyendo la elección de las muestras y la reevaluación del método de TL, un punto crítico.

\begin{abstract}
Radiocarbon and thermoluminiscense dates of the Late or Inca Horizon from South Central and Meridional andean areas supposed older than the usualy accepted "historic" chronology are contrasted with the chronology for the heart of the Empire independently proposed by Adamska and Micheczynski (1996) and by Bauer (1992) and supported by a statistical analysis of radiocarbon dates and considerations of archaeological nature. It is concluded that the chronology of the development of the Inca State is until now a problem that require more dates. The sample selection and the revaluation of the TL method is a critical point.
\end{abstract}

1 Sociedad Chilena de Arqueología, Email: museospa@entelchile.net Santiago.

\section{Introducción}

Esta comunicación se originó de algunas notas que llevaba al encuentro "Dominio Inca en las quebradas altas del Loa Superior" realizado en Caspana en el mes de agosto del año 1999, con el propósito de motivar una discusión en relación a este tema y en especial, a lo que concierne a la naturaleza e hitos cronológicos de la expansión inca hacia las áreas Centro Sur y Meridional andinas, por lo tanto, sólo debe considerarse como una puesta al día de la información publicada, disponible sobre el tema y con un final abierto.

Tradicionalmente la cronología del Estado inca se había fundamentado en hechos supuestamente históricos obtenidos de las relaciones de los cronistas y de testimonios oculares. Aunque la secuencia de los gobernantes incas proporcionada por la relación de Cabello de Valboa es motivo de discusión (Zuidema 1964, Rostworowski 1983). La cronología histórica más aceptada es la propuesta por Rowe (1944) que, en líneas generales, distingue una fase Pre Imperial que se iniciaría alrededor del 1200 DC y otra fase Imperial comprendida entre el 1438 y 1539 DC. La fecha inicial de esta segunda fase estaría determinada por el reinado del Inca Pachacuti bajo el cual se iniciaría la expansión imperial y, como corolario, después de esa fecha deberían datar los establecimientos incas en las áreas periféricas y la cerámica cuzqueña o sus imitaciones originadas en dichos territorios.

Esta interpretación "histórica" ha sido objeto de un creciente criticismo que argumenta que las crónicas no deben interpretarse literalmente, por cuanto ellas deberían leerse como representaciones 
míticas del pasado, destinadas a legitimar la desigualdad social por los núcleos de poder y que los cronistas dispusieron artificialmente en un orden cronológico (Urton 1992).

Aunque pareciera evidente que la arqueología se presentaba como una alternativa independiente para contrastar estos hechos y precisar su cronología, llama la atención la escasa investigación arqueológica sistematizada desarrollada en el núcleo del imperio y que sólo en fecha reciente se ha ido subsanando (Bauer 1992).

En lo que respecta a la cronología, la escasez hasta años recientes de fechas absolutas que delimitaran en el tiempo este desarrollo estatal y su posterior expansión hacia la periferia, pudo haber sido influida por la aceptación a priori por parte de los arqueólogos de la secuencia histórica, o que la imprecisión estadística de las fechas absolutas hacía poco viable su utilización para este período de tiempo relativamente reducido.

Algunas de estas fechas absolutas recientes, obtenidas de preferencia de localidades alejadas del núcleo central imperial, han arrojado fechas sorpresivamente más antiguas que las originalmente estimadas. Estos hallazgos aparentemente contradictorios han sido rechazados por algunos: Earle, Ravinés, Schiappacasse, o aceptados, en forma condicional por otros: Pärsinnen, Muñoz, Stehberg. Pärssinen (1997) citando a Braudel, hace notar acertadamente que los cambios políticos, sociales o religiosos no necesariamente producen cambios bruscos de la cultura material o viceversa.

Por haber sido las áreas periféricas correspondientes a Chile y Argentina dónde se originaron la mayoría de estas fechas supuestamente aberrantes, iniciaremos nuestro análisis desde la periferia hacia el centro.

\section{Bolivia}

De la localidad de Tiquischullpa, vecina a Caquiaviri, en el territorio Pacajes, se dispone de 6 fechas C14 calibradas y obtenidas con acelerador de masa provenientes de la excavación de una estructura erigida con técnica inca. Pärsinnen, (1997), a quién debemos esta información, anali- za estas fechas y la historia de la estructura, concluyendo que existe un sello carbonizado fechado entre los años 1310 y 1450 DC y que la fragmentación de alfarería incaica bajo dicho sello debe ser anterior a esa fecha. La opinión del autor es que los motivos inca de la cerámica del estrato más profundo corresponden a piezas de imitación local y que la cerámica cuzqueña tendría un origen más antiguo que el tradicionalmente aceptado. No descarta la posibilidad que algunos de esos motivos sean originarios de la cuenca del Titicaca, y hallan sido posteriormente asimilados por los incas a su iconografía.

\section{Extremo norte de Chile}

\section{Tarapacá}

Muñoz y Chacama (1988) y Muñoz y colaboradores (1997), han dado a conocer 36 fechas absolutas provenientes de sitios incaicos localizados en la sierra de Arica. Tres fechas son de $\mathrm{C} 14$ y las restantes de TL obtenidas de fragmentación estilo Saxamar o de imitaciones cuzqueñas. Del total de fechas, 28 oscilan entre 1410 a 1535 años DC, pero hay siete inusualmente tempranas entre el $1210 \mathrm{y}$ 1370 DC: tres pertenecen al tambo de Zapahuira y dos son del valle de Codpa (Tablas 1 y 2).

Con anterioridad, Schiappacasse y colaboradores (1991), en un análisis de 45 fechados de TL de cerámicas de los valles de Azapa y de Camarones que abarcan diferentes periodos de tiempo, siete de ellas correspondían al estilo Saxamar o a estilos provinciales con motivos cuzqueños. Tres fechas fueron anteriores al 1400 DC: dos del estilo Saxámar y la tercera de un fragmento de imitación cuzqueña que arrojó la determinación sorprendente de $1230 \pm 50$ DC. Es de notar que dos fragmentos cerámicos del mismo estilo y del mismo sitio mostraron una diferencia de alrededor de 100 años (Tabla 3).

\section{Antofagasta}

En la región de Antofagasta se dispone de una batería de fechas absolutas del sitio Pukara de Turi de la provincia de El Loa proporcionadas por Aldunate (1993) y por Varela y colaboradores (1993). Dos fechas de TL de 1400 y $1540 \pm 40$ años DC corresponden al estilo tardío San Pedro 
Tabla 1. Fechas TL (Muñoz y Chacama 1988, Muñoz et al. 1997)

\begin{tabular}{|c|c|c|c|}
\hline SECTOR & SITIO & ESTILO & FECHADO \\
\hline LIVILCAR & PUBRIZA & Saxamar & $1490 \pm 50 \mathrm{DC}$ \\
\hline \multirow{5}{*}{ ZAPAHUIRA } & ZAPAHUIRA 1 & Saxamar & $1240 \pm 75 \mathrm{DC}$ \\
\hline & ZAPAHUIRA 1 & Inca N/R Tardío & $1210 \pm 100 \mathrm{DC}$ \\
\hline & ZAPAHUIRA 2 & Inca Altiplánico & $1520 \pm 50 \mathrm{DC}$ \\
\hline & HUAYCUTA & Chilpe & $1430 \pm 50 \mathrm{DC}$ \\
\hline & CHAPICOLLO & $\mathrm{N} / \mathrm{R}$ & $1500 \pm 50 \mathrm{DC}$ \\
\hline \multirow[t]{2}{*}{ CHAPIQUIÑA } & LACO ALTO & Tumilaca & $1370 \pm 45 \mathrm{DC}$ \\
\hline & & N/R ¿Sillustani? & $1505 \pm 50 \mathrm{DC}$ \\
\hline \multirow[t]{14}{*}{ BELÉN -TICNAMAR } & HUAIHUARANI & Chilpe & $1480 \pm 60 \mathrm{DC}$ \\
\hline & HUAIHUARANI & San Miguel & $1440 \pm 60 \mathrm{DC}$ \\
\hline & HUAIHUARANI & $N / R$ & $1515 \pm 45 \mathrm{DC}$ \\
\hline & HUAIHUARANI & Charcollo & $1475 \pm 30 \mathrm{DC}$ \\
\hline & HUAIHUARANI & Engobado Rojo & $1510 \pm 35 \mathrm{DC}$ \\
\hline & INCAHULLO & Local sin decoración & $1470 \pm 60 \mathrm{DC}$ \\
\hline & TRIGALPAMPA & Engobado Rojo & $1485 \pm 60 \mathrm{DC}$ \\
\hline & ANCOPACHANE & Engobado Rojo & $1430 \pm 70 \mathrm{DC}$ \\
\hline & SAXAMAR & Chilpe & $1325 \pm 70 \mathrm{DC}$ \\
\hline & SAXAMAR & San Miguel & $1460 \pm 50 \mathrm{DC}$ \\
\hline & SAXAMAR & Charcollo & $1450 \pm 55 \mathrm{DC}$ \\
\hline & SAXAMAR & Rojo/Marrón & $1450 \pm 55 \mathrm{DC}$ \\
\hline & SAXAMAR & N/R Altiplánico 1 & $475 \pm 50 \mathrm{DC}$ \\
\hline & SAXAMAR & Gentilar & $1490 \pm 60 \mathrm{DC}$ \\
\hline \multirow[t]{3}{*}{ COBIJA } & COBIJA 1 & Inca Altiplánico & $1440 \pm 50 \mathrm{DC}$ \\
\hline & COBIJA 1 & Inca Altiplánico & $1410 \pm 60 \mathrm{DC}$ \\
\hline & COBIJA 2 & Inca Cuzqueño & $1480 \pm 50 \mathrm{DC}$ \\
\hline \multirow[t]{4}{*}{ CODPA } & INCAUTA & Chilpe & $1280 \pm 60 \mathrm{DC}$ \\
\hline & MOLLE GRANDE 1 & Local N/R & $1410 \pm 70 \mathrm{DC}$ \\
\hline & MOLLE GRANDE 2 & Inca & $1370 \pm 60 \mathrm{DC}$ \\
\hline & CACHICOCA & Saxamar & $1320 \pm 50 \mathrm{DC}$ \\
\hline \multirow[t]{2}{*}{ MIÑITA } & MIÑITA 4B & Saxamar & $1525 \pm 50 \mathrm{DC}$ \\
\hline & MIÑITA 4B & Saxamar & $1525 \pm 50 \mathrm{DC}$ \\
\hline \multirow[t]{3}{*}{ PUNA } & TAMBO CHUNGARA & N/R & $1605 \pm 30 \mathrm{DC}$ \\
\hline & TAMBO TACORA & Inca Local & $1590 \pm 40 \mathrm{DC}$ \\
\hline & TAMBO CAQUENA & N/R & $1535 \pm 30 \mathrm{DC}$ \\
\hline
\end{tabular}


Tabla 2. Fechas C14 (Muñoz y Chacama 1988, Muñoz et al. 1997)

\begin{tabular}{llc}
\hline SECTOR & \multicolumn{1}{c}{ SITIO } & FECHADO \\
\hline ZAPAHUIRA & ZAPAHUIRA. CHULLPA 1 & $1415 \pm 95$ DC \\
& ZAPAHUIRA. CHULLPA 2 & $1290 \pm 60$ DC \\
& TAMBO 1 R. 6 & $1515 \pm 105$ DC \\
\hline
\end{tabular}

Tabla 3. Fechas TL (Schiappacasse et al. 1991)

\begin{tabular}{lll}
\hline SITIO & ESTILO & FECHADO \\
\hline POBLACION CALETA CAMARONES NORTE & Inca No Decorado & $1420 \pm 30 \mathrm{DC}$ \\
HACIENDA CAMARONES & Saxamar & $1320 \pm 90 \mathrm{DC}$ \\
PACHICA & Inca Altiplánico & $1530 \pm 60 \mathrm{DC}$ \\
SAGUARA & Imitación Cuzco & $1230 \pm 50 \mathrm{DC}$ \\
SAGUARA & Saxamar & $1350 \pm 80 \mathrm{DC}$ \\
SAGUARA & Inca Altiplánico & $1520 \pm 80 \mathrm{DC}$ \\
SAGUARA & Inca Altiplánico & $1560 \pm 35 \mathrm{DC}$ \\
& & \\
\hline
\end{tabular}

Rojo Violáceo o Grupo 38 Rojo Pintado, y otra de 1530 años DC a una escudilla ornitomorfa inca.

Cuatro grupos cerámicos de situación cronológica desconocida proporcionaron fechas tardías: Rojo Alisado Grupo 1: 1490 años; Rojo Burdo Grupo 2: 1450 y $1560 \pm 60$ DC; Grupo 31 B: 1420 DC y Grupo 37: 1480 años DC. También proporcionaron fechas tardías inusuales algunos grupos cerámicos de edad pre Tardía ya establecida: el Grupo 37 Rojo Pulido $1480 \pm 70$ años DC, el Grupo 9B variedad Ayquina 1470 años DC y el Grupo 32, variedad Dupont: 1430 años DC.

Para nuestro propósito hay además dos fechas de C14: una de la Estructura 500 B-7 de $1540 \pm 80$ años DC (calibr. 50\% 1500 años) asociada a una vasija Rojo Pintada y otra de la Estructura 56 F4/ 5 o kallanka de $1420 \pm 80$ años DC (calibr. 1390 años DC); la muestra de TL de $1540 \pm 40$ años corresponde al mismo estrato.

\section{Norte Chico}

\section{Copiapó}

Del sitio tardío Punta Brava del río Copiapó, Castillo (1999) proporciona dos fechas de C14 de 1260 \pm 50 y $1440 \pm 50$ años DC obtenidas de basuras domésticas, la primera posiblemente está fechando una ocupación pre inca.

De diferentes sepulturas del cementerio del sitio Iglesia Colorada en el río Pulido, afluente del Copiapó, el mismo autor enumera seis fechas de TL que van del 1100 al 1475 años DC. No se precisan las características de la cerámica fechada, pero se señala que ninguna de las tumbas tenía piezas de origen inca. 


\begin{tabular}{ll}
\hline SITIO & FECHADO \\
\hline IGLESIA COLORADA & $1100 \pm 70 \mathrm{DC}$ \\
IGLESIA COLORADA & $1230 \pm 90 \mathrm{DC}$ \\
IGLESIA COLORADA & $1300 \pm 70 \mathrm{DC}$ \\
IGLESIA COLORADA & $1315 \pm 70 \mathrm{DC}$ \\
IGLESIA COLORADA & $1420 \pm 60 \mathrm{DC}$ \\
IGLESIA COLORADA & $1475 \pm 50 \mathrm{DC}$ \\
\hline
\end{tabular}

Dos de estas fechas son sincrónicas con otra fecha de TL del sitio Altos Blancos, en el río Del Potro, de $1350 \pm 55$ años DC, que fecha un momento pre inca correspondiente a la cultura Copiapó.

Stehberg (1991), resume los fechados absolutos obtenidos de sitios inca estudiados por él, localizados en el Norte Chico: tres fechas son de C14 y dos de TL. Hace mención además de algunas fechas de Chile central cuyo detalle veremos en su oportunidad, basándonos en las comunicaciones originales aparecidas con fecha posterior al artículo de Stehberg. Enumeraremos estas fechas en relación a las cuencas fluviales de norte a sur intercalando otras fechas dadas a conocer por otros autores.

Huasco

Fechas de $\mathrm{C} 14$ de sitios con características incas: Tambo Pasteadero $1240 \pm 90$ años DC, muestra de fogón asociada a cerámica Inca provincial; Tambo Laguna Chica $1370 \pm 80$ años DC asociada a cerámica Inca-Diaguita y del Tambo Colinai $1490 \pm 80$ años DC no asociada a cerámica. Es de notar que fracciones de las mismas muestras procesadas por otro laboratorio fueron desechadas "por lo disparatado de los resultados."

\section{Elqui}

Se conocen dos fechas de TL de $1520 \pm 50$ años y de $1510 \pm 60$ años DC pertenecientes a fragmentos cerámicos de la Fase 3 Inca-Diaguita y otras dos fechas de $1430 \pm 50$ y $1410 \pm 60$ años DC de la cerámica Diaguita Fase 2 (Suárez y cols. Ms 1988 citada por Cornejo 1989).

Rodríguez y cols. (1993) también comunican fe- chas de TL correspondientes a fragmentos de cerámica Inca-Diaguita del sitio Fundo Coquimbo del río Elqui pero de edad dispar de $1200 \pm 90$ años y $1260 \pm 70$ años DC.

\section{Limarí}

Del sitio Planta Pisco Control de atribución inca, Rodríguez y cols. (1993) proporcionan las fechas de TL de $1205 \pm 80$ años y $1375 \pm 60$ años DC.

\section{Choapa}

Se conocen dos fechas de TL del sitio Conchuca comunicadas por Stehberg (1991), de $1450 \pm 60 \mathrm{y}$ $1660 \pm 60$ años DC.

\section{Chile Central}

\section{Aconcagua}

Del sitio Cerro La Cruz, Rodríguez y cols. (1993), han obtenido cuatro fechas TL de $1285 \pm 70,1390$ $\pm 70,1430 \pm 60$ y $1430 \pm 70$ años DC. Todos los fragmentos fechados corresponden al estilo IncaDiaguita, exceptuando el primero que es de cerámica no decorada.

Durán y cols. (1993), comunican una fecha TL de un fragmento de aríbalo de $950 \pm 120$ años DC del sitio Rinconada El Coligüe en la Cuesta de Chacabuco. Esta fecha es inusual porque es más antigua que la de un fragmento cerámico Aconcagua Negro sobre Salmón del período pre inca con fecha de $1000 \pm 100$ años DC perteneciente al mismo sitio.

También llama la atención la disparidad de la fecha de TL con la de C14 del sitio La Victoria: un fragmento de cerámica del nivel $10-20 \mathrm{~cm}$ de la excavación fechó $940 \pm 130$ años DC en circunstancia que el mismo nivel proporcionó la fecha de C14 de $1460 \pm 50$ años DC.

\section{Cachapoal}

Planella y cols. (1993) obtuvieron tres fechados por TL del sitio Cerro Grande de la Compañía: $1430 \pm 60$ años DC de un fragmento de aríbalo, $1480 \pm 60$ años DC de un estilo no identificado y $1530 \pm 45$ años DC de un plato inca. 
Una fecha de TL de $1365 \pm$ años DC de un sitio en el río Claro que había sido atribuida anteriormente al periodo inca corresponde en realidad al estilo Viluco de ubicación pre inca según Cáceres y cols. (1993).

\section{Argentina}

Stehberg (1991) enumera además 11 fechas de C14 obtenidas de sitios considerados incas en el territorio argentino:

\begin{tabular}{lc}
\hline SITIO & FECHA $\left(^{*}\right)$ \\
\hline LA HUERTA & $1370 \mathrm{DC}$ \\
LA HUERTA & $1410 \mathrm{DC}$ \\
NEGRO OVERO & $1380+/-80 \mathrm{DC}$ \\
CERRO TORTOLAS & $950 \mathrm{DC}$ \\
CIENAGA DE YALGUARAZ & $1465+/-60 \mathrm{DC}$ \\
CIENAGA DE YALGUARAZ & $1530+/-60 \mathrm{DC}$ \\
CIENAGA DE YALGUARAZ & $1560+/-90 \mathrm{DC}$ \\
TAMBO DE TAMBILLOS & $1540+/-70 \mathrm{DC}$ \\
CERRO COLORADO & $1000+/-10 \mathrm{DC}$ \\
TOKOTA & $1525+/-80 \mathrm{DC}$ \\
MERCEDARIO & $1600 \mathrm{DC}$ \\
MERCEDARIO & $1560 \mathrm{DC}$ \\
\hline
\end{tabular}

$\left.{ }^{*}\right)$ algunas fechas están incompletas.

En resumen y sin eliminar algunas de las fechas que parecieran datar contextos de períodos más antiguos, se dispone de un total de 80 fechas de sitios localizados en el territorio meridional del imperio, 21 de las cuales anteceden al año 1400 de la era y corresponden al $26 \%$ de la muestra. Hemos escogido el límite de 1400 años por consideraciones que se analizarán más adelante.

\section{Fechados adicionales}

Adamska y Micheczynski (1996) de la Universidad de Varsovia, analizan un conjunto de 46 fechas absolutas de $\mathrm{C} 14$ atribuidas al período inca, por su asociación a estructuras arquitectónicas o de contextos culturales reconocidos como incas. Además de 43 fechas del territorio peruano, incluyen dos fechas del Ecuador, una de Argentina y dos de Chile. Como se dijo, todas las fechas son de $\mathrm{C} 14$ menos las de Chile que corresponden a las fechas de TL: UCTL 231 y 229 del Cerro Grande de la Compañía. Por la cita bibliográfica, la información la obtuvieron de Dillehay (1992) donde equivocadamente las fechas se presentan como obtenidas por radiocarbono. Además, no incluyen las fechas más recientes de Bolivia y de Chile que se analizaron en párrafos anteriores pero sí consideran las fechas, algunas tempranas, de la Costa Central y de Arequipa comunicadas por Ravinés.

Los autores utilizan las curvas de calibración de Stuiver y colaboradores (1993), y señalan que habitualmente, debido a la dispersión del conteo, se utiliza la media y la SD como la mejor aproximación de la edad, pero asumiendo una curva normal. La presencia de diferentes peaks en la curva de calibración radiocarbónica que puede ocurrir en algunos fechados, no justifica atribuir una correspondencia unívoca entre la edad de C14 obtenida y la edad calendárica. A fin de subsanar este inconveniente proponen realizar una calibración probabilística de las fechas radiocarbónicas, consistente en derivar de la curva de distribución estadística radiocarbónica otra curva probabilística de la edad calendárica. Esta última curva puede adoptar varias formas: desde una única curva gaussiana a formas complejas, con diferentes peaks, correspondiendo en este caso la edad más probable a la representada por la curva que abarca un área mayor. Si no hubiere una diferencia estadística significativa entre las áreas, dicha muestra debería considerarse de fecha indeterminada.

En el estudio se agruparon las muestras atribuidas a las fases pre imperial, asociada a cerámica Killque o a construcciones con técnica de pirca, y a la Imperial, asociada a cerámica cuzqueña o a construcciones de masonería. Concluyen su estudio estadístico estableciendo que la mejor estimación de las fechas calendáricas para el estado Inca serían: fase pre imperial del 1280 al 1396 DC y fase imperial del 1401 al 1518 DC.

La curva de las fechas correspondientes al período imperial es compleja con tres peaks en 1325 , 1440 y 1600 abarcando un área mayor la curva central.

Bauer (1992), por su parte, tomando en consideración las fechas disponibles de la región cuzqueña, también establece como más probable una antiguiedad del 1260 al 1390 DC para el período Killque o pre imperial y el año 1400 DC para el inicio del período imperial. Deduce ade- 
más que la cerámica tipo Cuzco y la arquitectura "cuzqueña" no son sincrónicas, antecediendo la arquitectura a la cerámica.

\section{Conclusión}

De lo expuesto puede concluirse que los resultados de un análisis estadístico de un número importante de 46 fechas radiocarbónicas, que abarca la mayor parte del imperio inca, junto a otras consideraciones de naturaleza arqueológica, son compatibles con la hipótesis planteada por el método histórico, aceptando una corrección de 30 años de la fecha propuesta. Sin embargo de un total aproximado de 80 fechas de TL y de C14 provenientes de las áreas Centro-Sur y Meridional andinas hay un número no despreciable de fechas que datan contextos o especímenes atribuidos al inca anteriores al 1400. Aunque algunas de ellas son discutibles por su excesiva antiguiedad o por estar en contradicción con otras evidencias del mismo contexto, queda un remanente que no puede ser desechado a priori, lo que obliga a tener en consideración otras hipótesis alternativas que proponen una mayor antigüedad para la fase imperial y para las cerámicas cuzqueña y derivadas.

La cronología absoluta del Estado Inca debe considerarse, entonces, como un problema no resuelto, abierto a la discusión, siendo necesarios mayores antecedentes para intentar esclarecerlo. Se hace indispensable disponer de una mayor cantidad de fechas absolutas obtenidas de muestras orgánicas de contextos incas bien definidos, evitando asociaciones espúreas por depositación secundaria o datar especímenes de clara filiación inca.

Por problemas de costo, en nuestro medio se ha recurrido de preferencia al método de TL, procedimiento que tendría la ventaja de fechar directamente el artefacto cultural. Considerando que su utilización se va a acrecentar en el futuro, sería oportuno realizar una revaluación crítica de todo el procedimiento desde sus etapas iniciales de toma de las muestras, colocación de los dosímetros, métodos de cálculo de la dosis radioactiva, etc. con el fin de minimizar el error experimental.

\section{Post Scriptum}

Con posterioridad a la redacción del artículo, el autor tuvo conocimiento de la comunicación de V.I. Williams y T.N. D'Altro y "El sur del Tawantinsuyu: Un dominio selectivamente intenso" publicada en Tawantinsuyu 5:170-178 (1998). Allí, además de un excelente análisis sobre la naturaleza de la ocupación inca en un sector del NOA, incide en el tema de la cronología, aportando 13 fechas $\mathrm{C} 14$ que sugieren una mayor antigüedad de lo tradicionalmente aceptado para el inicio de la expansión imperial.

\section{BIBLIOGRAFIA}

ADAMSKA, A. y A. MICHECZYNSKI. Towards radiocarbon 1996 chronology of de inca state. Boletín de la misión arqueológica andina 1: 35 58, Varsovia.

ALDUNATE, C. Arqueología del Pukara de Turi. Actas 1993 del XII Congreso Nacional de Arqueología Chilena. Boletín del Museo Regional de la Araucanía. Tomo II, $\mathrm{N}^{\circ} 4$ : 61-78, Temuco.

BAUER, S. The development of the inca state. 1992 University of Texas Press, Austin.

CACERES, I., E. ASPILLAGA, A. DEZA y A. ROMAN. Un 1993 sitio agroalfarero tardío en la cuenca del río Cachapoal, Chile Central. Actas del XII Congreso Nacional de Arqueolo- gía Chilena. Boletín Museo Regional de la Araucanía, tomo II, $\mathrm{N}^{\circ} 4: 423-428$, Temuco.

CASTILLO, G. Los períodos Intermedio Tardío y Tar1997 dío: Desde la Cultura Copiapó al dominio Inca. En Culturas prehistóricas de Copiapó, Niemeyer, Cervellino y Castillo (Eds.), pp. 163-282, Museo Regional de Copiapó.

CORNEJO, L. El plato zoomorfo diaguita: Variabilidad 1989 y especificidad. Boletín del Museo Chileno de Arte Precolombino 3:47-80, Santiago.

DILLEHAY, T. Current Research. American Antiquity $1992 \quad 57: 163$. 
DURAN, E., A. RODRIGUEZ, y C. GONZALEZ. Sistemas 1993 adaptativos de poblaciones prehispánicas en el Cordón de Chacabuco. Actas del XII Congreso Nacional de Arqueología Chilena. Boletín Museo Regional de la Araucanía. Tomo II. $\mathrm{N}^{\circ} 4$ : 235-247, Temuco.

MUÑOZ, I. y J. CHACAMA. Cronología por termo luminis 1988 cencia para los periodos Intermedio Tardío y Tardío en la sierra de Arica. Chungara 20: 19-45, Arica.

MUÑOZ, I., J. CHACAMA y M. SANTOS. Tambos, pukaras 1997 y aldeas, evidencias del poblamiento humano prehispánico tardío y de contacto indígena-europeo en el extremo norte de Chile. Análisis de los patrones habitacionales y de nuevas dataciones radiométricas. Diálogo Andino 16: 123190, Arica.

PÄRSSINEN, M. y A. SIIRIÄINEN. Inca-style ceramics and 1997 their chronological relationship to the inca expansion in the southern lake Titicaca area ( Bolivia). Latin American Antiquity 8, $\mathrm{N}^{\circ} 3: 255-272$.

PLANELLA, M., STEHBERG. R., TAGLE, B., NIEMEYER, 1993 H. y DEL RIO, C. La fortaleza indígena del Cerro Grande de la Compañía (Valle del Cachapoal ) y su relación con el proceso expansivo meridional incaico. Actas del XII Congreso Nacional de Arqueología Chilena. Boletín Museo Regional de la Araucanía, Tomo II, $\mathrm{N}^{\circ}$ 4: 403-421, Temuco.

RODRIGUEZ, A, R. MORALES, C. GONZALEZ y D. JACK1993 SON. Cerro La Cruz: Un enclave económico y administrativo incaico. Actas del XII Congreso Nacional de Arqueología Chilena. Boletín Museo Regional de la Araucania, Tomo II, N4: 201-222, Temuco.

ROSTWOROSKI, M.La diarquía entre los incas. Estructu1983 ras andinas del poder, pp. 130-174, IEP, Lima.

ROWE J. An introduction to the archaeology of 1944 Cuzco. Papers of the Peabody Museum of American archaelogy and ethnology 27:2, Harvard University, Cambridge.

SCHIAPPACASSE, V., A. ROMAN, I. MUÑOZ, A. DEZA, y 1991 G. FOCACCI. Cronología por termoluminiscencia de la cerámica del extremo norte de Chile. Actas del XI Congreso Nacional de Arqueología Chilena.pp. 43-59, Santiago.

STEHBERG, R. El límite inferior cronológico de la ex1991 pansión incaica en Chile. Xama 4-5: 63 89.

STUIVER, M., A. LONG y R.S. KRA (Eds.) Calibration 1993. 1993 Radiocarbon 35 (1): 1-224.

URTON, G. Foreword. The development of the In1992 ca State. Bauer (Ed.), University of Texas Press, Austin.

VARELA, V., M. URIBE y L. ADAN La cerámica arqueoló1999 gica del sitio 02-TU-001: "Pukara" de Turi. Boletín del Museo Regional de La Araucanía 4:107-122, Temuco.

ZUIDEMA R. The ceque system of Cuzco.The social 1964 organization of the capital of the incas. Leiden E. J. Brill, Leiden. 